\title{
Inhomogeneous Markov Models for Describing Driving Patterns
}

\author{
Iversen, Emil Banning; Møller, Jan K.; Morales, Juan Miguel; Madsen, Henrik
}

Published in:

IEEE Transactions on Smart Grid

Link to article, DOI:

10.1109/TSG.2016.2520661

Publication date:

2017

Document Version

Peer reviewed version

Link back to DTU Orbit

Citation (APA):

Iversen, E. B., Møller, J. K., Morales, J. M., \& Madsen, H. (2017). Inhomogeneous Markov Models for Describing Driving Patterns. IEEE Transactions on Smart Grid, 8(2), 581-588. [7414468].

https://doi.org/10.1109/TSG.2016.2520661

\section{General rights}

Copyright and moral rights for the publications made accessible in the public portal are retained by the authors and/or other copyright owners and it is a condition of accessing publications that users recognise and abide by the legal requirements associated with these rights.

- Users may download and print one copy of any publication from the public portal for the purpose of private study or research.

- You may not further distribute the material or use it for any profit-making activity or commercial gain

- You may freely distribute the URL identifying the publication in the public portal

If you believe that this document breaches copyright please contact us providing details, and we will remove access to the work immediately and investigate your claim 


\title{
Inhomogeneous Markov Models for Describing Driving Patterns
}

\author{
Emil B. Iversen, Jan K. Møller, Juan M. Morales, Member, IEEE, and Henrik Madsen
}

\begin{abstract}
It has been predicted that electric vehicles will play a crucial role in incorporating a large renewable component in the energy sector. If electric vehicles are integrated in a naive way, they may exacerbate issues related to peak demand and transmission capacity limits while not reducing polluting emissions. Optimizing the charging of electric vehicles is paramount for their successful integration. This paper presents a model to describe the driving patterns of electric vehicles, in order to provide primary input information to any mathematical programming model for optimal charging. Specifically, an inhomogeneous Markov model that captures the diurnal variation in the use of a vehicle is presented. The model is defined by the time-varying probabilities of starting and ending a trip and is justified due to the uncertainty associated with the use of the vehicle. The model is fitted to data collected from the actual utilization of a vehicle. Inhomogeneous Markov models imply a large number of parameters. The number of parameters in the proposed model is reduced using $B$-splines.
\end{abstract}

Index Terms-B-splines, Driving patterns, Electric vehicles, Inhomogeneous Markov chain, Hidden Markov model

\section{INTRODUCTION}

Electric vehicles (EVs) have no emissions and are a sustainable alternative to conventional vehicles, provided that the energy used for charging is generated by renewable sources. Electricity generation from renewable energy sources, such as wind, solar and wave energy depends on weather conditions and is inherently uncertain. In the absence of a large-scale infrastructure for energy storage, electricity has to be consumed as it is produced. EVs may help overcome this by charging when energy from renewable sources is abundant and by supplying power into the electrical grid at times of high demand. With electricity from renewable sources, EVs represent a sustainable zero-emissions alternative to conventional fossil-fuel-based vehicles.

On the contrary, if the fleet of EVs are charged in a naive way, it may increase the peak electricity demand. As a consequence, the extra energy needs will be covered by peaksupply units, nullifying the decrease in emissions gained from switching to EVs. At the same time the transmission grid is strained. As the the fleet of EVs must necessarily be charged as individual vehicles, an understanding of the availability and user needs at an individual level becomes fundamental. The impact of EVs on the power grid has been considered in several studies ([1], [2]). Without scheduling algorithms, EVs have the potential to cause imbalances between power demand and supply. In [3], [4], however, these effects can be mitigated by scheduling and the amount of vehicles that can be introduced without infrastructure investments can be significantly increased and curtailing consumption may in- crease social welfare. In [5] different charging schemes are considered.

In order to adequately characterize the smart grid, the aggregate power demand and flexibility should be described. To achieve this the aggregate demand and flexibility of different demand side components should be modeled, such as the fleet of electric vehicles and their associated charging. In order to accurately model an electric vehicle fleet the understanding of single vehicle behavior is required as every single vehicle does not have the same characteristics. Thus describing user behavior is one of fundamental building blocks in the development of the smart grid ([6], [7], [8]). This is particularly the case when considering things as autonomous demand side management or indirect control ([9], [10]).

As EVs primary purpose is transportation, not energy storage, it is essential to charge each vehicle to have enough energy to cover desired trips. Thus a model for capturing the utilization of a single vehicle is essential for the efficient operation of each vehicle and the EV fleet in general.

In the technical literature, albeit observed vehicle usage has been considered ([11], [12]), the stochastic modeling of the use of a single vehicle has received little attention ([13]). Rather, the scientific community has focused on both the analysis of the impact of charging EVs and when to charge EVs ([14]). Large-scale integration of EVs into the power grid has been studied in several papers, ([15], [16], [17], [18], [19]). Peak load, charging strategies, network losses, costs and market equilibrium strategies have been considered.

Inhomogeneous Markov chains have previously been used in conjection with EVs. A number of authors employ this approach to simulate the utilization of a population of EVs to model the total electricity demand of the fleet of EVs ([20], [21], [22], [23], [24]). Due to practical and methodological issues, these works prove too coarse with respect to capturing the use of a single vehicle. They are based on the assumption that the same underlying stochastic process generates the driving patterns of all the vehicles in the population. While this approximation may be accurate enough for applications where the aggregate behavior of the EV fleet is needed, it is not detailed enough for applications where the modeling of the driving patterns of a specific vehicle is required. The stochastic dynamics of the utilization of a particular vehicle can be very different from the dynamics of the population. Furthermore, modeling the driving behavior of a single vehicle poses nontrivial challenges related to limited data, validity of the Markov assumption, and time resolution, all of which are addressed in this paper. However, Markov models have been used to simulate the utilization of a single vehicle while driving, focusing on trip duration, consumption, speed and 
type-of-use ([25]) and on road network dynamics particularly for electric vehicle applications ([26]).

This paper bridges the gap between these two approaches. It presents a genuine single vehicle model that can be easily exploited, for example, by decision-making tools for charging an $\mathrm{EV}([27])$. It provides advances in modeling driving patterns and does not rely on typical, average or stylized use of a vehicle. The model is fitted to a specific vehicle based on observed data from the utilization of that vehicle. An inhomogeneous Markov model is applied to capture the diurnal variation of the driving pattern. These types of models have a large number of parameters to be estimated. A generalized linear model is then applied to substantially reduce this number, under the constraint of limited data and the need for a high temporal resolution. We use hidden Markov models to adequately capture the duration of the trips. Furthermore, the model satisfies the Markov property, which is an important aspect for decision making as the planning problem can be solved using only present knowledge thus not relying on future knowledge. The proposed model thus provides several benefits over homogeneous Markov chain models, inhomogeneous Markov models without parameter reductions and nonparametric approaches. Further, it does not rely on assumptions regarding the use of the vehicle, and consequently a versatile model is obtained. Applying the model within a stochastic optimization framework will allow for capturing issues related to charging, availability, and costs of using an EV ([27]).

The literature on electric vehicles and their integration into the smart grid is now vast. For an in-depth review the reader is referred to one of the recent survey papers investigating this topic ([28], [29], [30]).

The paper is organized as follows: Section 2 gives a brief introduction to inhomogeneous Markov chains. In Section 3 the number of parameters in the model is reduced by applying B-splines to a generalized linear model. Section 4 provides a numerical example of the model. Section 5 concludes and provides directions for future research.

\section{An Inhomogeneous Markov Chain}

A state-space approach is proposed to describe the use of a vehicle. This approach models the vehicle as being in one of several distinct states. In its simplest form the model has two states, which capture whether the vehicle is either driving or not driving. A more extensive model may include information about where the vehicle is parked, where it is driving, or what type of trip the vehicle is on. In this section we start from a general state-space approach and finish with a detailed description of the two-state model.

\section{A. Discrete Time}

Consider a sequence $X$ of random variables $X_{t}, t \in$ $\{0,1,2, \ldots\}$, which take on values in the countable set $S$, referred to as the state space. A Markov chain is a random process where future states, conditioned on the present state, do not depend on the past states. We use the definition and notation from [31].
A Markov chain is uniquely characterized by the transition probabilities from state $j$ to state $k$, i.e.

$$
p_{j k}(t)=\mathbb{P}\left(X_{t+1}=k \mid X_{t}=j\right) .
$$

If the transition probabilities do not depend on $t$, it is called a homogeneous Markov chain. If the transition probabilities depend on $t$, it is known as an inhomogeneous Markov chain.

In order to estimate parameters in an inhomogeneous Markov chain an assumption of periodicity is needed. The case for longer periods is that they may better capture the nature of the use of the vehicle. A model with weekly periodicity would allow for capturing different behavior on different weekdays. However, using longer periods reduces the effective data available (by reducing the number of effective periods in the data set). Further, it increases the number of parameters in the model. We choose a period length of one day. However, the procedure outlined is not specific to this choice and can be repeated with other period lengths.

Considering the use of a vehicle we assume that the probability of a transition from state $j$ to state $k$ on any specific weekday is the same. Furthermore, we assume that the transition probabilities are the same on all weekdays, that is, from Mondays to Fridays. If the sampling time is in minutes, this leads to the assumption:

$$
p_{j k}(t)=p_{j k}(t+1440),
$$

where 1440 is the number of minutes in a day. In other words the transition probabilities, defined by (1), are constrained to be a function of the time $s$ in minutes in the diurnal cycle. The matrix containing the transition probabilities is given by

$$
\mathbf{P}(s)=\left(\begin{array}{cccc}
p_{11}(s) & p_{12}(s) & \ldots & p_{1 N}(s) \\
p_{21}(s) & p_{22}(s) & \ldots & p_{2 N}(s) \\
\vdots & \vdots & \ddots & \vdots \\
p_{N 1}(s) & p_{N 2}(s) & \ldots & p_{N N}(s)
\end{array}\right)
$$

where $p_{j j}(s)=1-\sum_{i=1, i \neq j}^{N} p_{j i}$.

With a time resolution in minutes, $s \in\{1,2, \ldots, 1440\}$. It follows that the conditional likelihood function, for the model with $N$ states, is given by ([32]):

$$
L(\mathbf{P}(1), \mathbf{P}(2), \ldots, \mathbf{P}(1440))=\prod_{s=1}^{1440} \prod_{j=1}^{N} \prod_{k=1}^{N} p_{j k}(s)^{n_{j k}(s)}
$$

where $n_{j k}(s)$ is the number of observed transitions from state $j$ at time $s$ to state $k$ at time $s+1$.

From the conditional likelihood function the maximumlikelihood estimate of $p_{j k}(s)$ can be found as:

$$
\widehat{p}_{j k}(s)=\frac{n_{j k}(s)}{\sum_{k=1}^{N} n_{j k}(s)} .
$$

A discrete time Markov model can be formulated based on the estimates of $\mathbf{P}(1), \mathbf{P}(2), \ldots, \mathbf{P}(1440)$. One apparent disadvantage of such a discrete time model is the huge number of parameters, namely $N \cdot(N-1) \cdot 1440$, where $N \cdot(N-1)$ parameters have to be estimated for each time step. Needless to say, the number of parameters to be estimated increases as the number of states increases. Another problem is linked to 
the number of observations, i.e. if $\sum_{k=1}^{N} n_{j k}\left(s^{\prime}\right)=0$ for some $s^{\prime}$, then $\widehat{p}_{j k}(s)$ is undefined.

A reduction in parameters may be obtained if the diurnal variation is negligible for some transitions, i.e. $p_{j k}(s)$ does not depend on $s$ for some pair $\{j, k\}$. Another way to reduce the parameters is to increase the time between samples. If the sampling time is every 10 minutes, the number of parameters would decrease to $N \cdot(N-1) \cdot 144$. This approach is a bit coarse and the number of parameters is still large. Besides, if another parameter reduction technique is subsequently applied to the data, information is lost compared to directly applying the technique to the data with a sampling time in minutes.

In the model with only two states, namely driving and not driving, the one-minute transition probability matrix becomes:

$$
\begin{aligned}
\mathbf{P}(s) & =\left(\begin{array}{cc}
p_{11}(s) & p_{12}(s) \\
p_{21}(s) & p_{22}(s)
\end{array}\right) \\
& =\left(\begin{array}{cc}
1-p_{12}(s) & p_{12}(s) \\
p_{21}(s) & 1-p_{21}(s)
\end{array}\right) .
\end{aligned}
$$

The number of parameters is then $2 \cdot 1440$. Assuming that the duration of the trip does not depend on the time of the day, i.e. $p_{21}(s)=p_{21}$, (with 2 being "driving" and 1 "not driving") the number of parameters is reduced to $1440+1$. Note that, as a result of this reduction, the duration of a trip is captured by a single parameter.

It follows that the conditional likelihood function, for the model with two states, is given by:

$$
L(\mathbf{P}(1), \mathbf{P}(2), \ldots, \mathbf{P}(1440))=\prod_{s=1}^{1440} \prod_{j=1}^{2} \prod_{k=1}^{2} p_{j k}(s)^{n_{j k}(s)},
$$

and the maximum-likelihood estimate $\hat{p}_{j k}(s)$ is computed from (5).

\section{B. Continuous Time}

The continuous time analog to the discrete time inhomogeneous Markov chain is presented below. The continuous time version provides a parameter reduction over the discrete time version, if certain structures are present and can be identified. Specifically, if the number of states is larger than two and it is impossible to switch directly between certain pairs of states, the continuous time variant will lead to a parameter reduction. Hence, if such structures are present, the continuous time variant is preferred over the discrete time model. To introduce the continuous time inhomogeneous Markov chain, we define ([31]):

$$
p_{j k}(t, u)=\mathbb{P}(X(u)=k \mid X(t)=j),
$$

where $t<u$. The model is based on the following assumptions when $\Delta u \rightarrow 0$ :

$$
\begin{aligned}
& p_{j j}(u, u+\Delta u)=1-q_{j j}(u) \Delta u+o(\Delta u) \\
& p_{j k}(u, u+\Delta u)=q_{j k}(u) \Delta u+o(\Delta u) \quad \forall j \neq k,
\end{aligned}
$$

also $0 \leq q_{j j}(u)<\infty$ and $0 \leq q_{j k}(u)<\infty$. The $q_{j k}(u)$ 's are known as the transition intensities. These assumptions lead to Kolmogorov's forward differential equation for inhomogeneous Markov processes, expressed in matrix notation as:

$$
\frac{\partial \mathbf{P}(t, u)}{\partial u}=\mathbf{P}(t, u) \mathbf{Q}(u)
$$

where $\mathbf{P}(t, u)=\left\{p_{j k}(t, u)\right\}$, i.e. $\mathbf{P}(t, u)$ is the matrix containing the $p_{j k}(t, u)$ 's. The matrix of transition intensities then becomes:

$$
\mathbf{Q}(u)=\left(\begin{array}{cccc}
-q_{11}(u) & q_{12}(u) & \ldots & q_{1 N}(u) \\
q_{21}(u) & -q_{22}(u) & \ldots & q_{2 N}(u) \\
\vdots & \vdots & \ddots & \vdots \\
q_{N 1}(u) & q_{N 2}(u) & \ldots & -q_{N N}(u)
\end{array}\right)
$$

Since $\sum_{k=1}^{N} p_{j k}(u, u+\Delta u)=1$, it follows from (9)-(10) that $\sum_{k=1}^{N} q_{j k}(u)=0 \forall j$, i.e. $q_{j j}(u)=$ $\sum_{k=1, k \neq j}^{N}-q_{j k}(u) \forall j$.

A simple Kolmogorov's differential equation is obtained if $\mathbf{Q}(t)$ is constant in the period $[t, t+T]$ :

$$
\mathbf{P}(t, t+T)=e^{\mathbf{Q}(t) T} \mathbf{P}(t, t)=e^{\mathbf{Q}(t) T},
$$

where $\mathbf{P}(t, t)$ contains the probability of moving between the different states between $t$ and $t$, i.e. in zero time, which is a matrix with ones on the diagonal and zero everywhere else. Suppose that $T=1$. Then the one minute transition probabilities are given by:

$$
\mathbf{P}(t, t+1)=\mathbf{P}(t)=e^{\mathbf{Q}(t)},
$$

where $\mathbf{P}(t)$ is the standard transition probability matrix for a discrete time Markov chain. If the model has two states, the matrix of transition intensities becomes:

$$
\mathbf{Q}(u)=\left(\begin{array}{cc}
-q_{11}(u) & q_{12}(u) \\
q_{21}(u) & -q_{22}(u)
\end{array}\right)=\left(\begin{array}{cc}
-q_{12}(u) & q_{12}(u) \\
q_{21}(u) & -q_{21}(u)
\end{array}\right)
$$

As mentioned previously, a continuous time Markov chain will allow for a parameter reduction if certain structures are present. Furthermore, identifying such structures will make the model more theoretically tractable. As a simple illustration of such a model, consider the case where there are four states, i.e. $N=4$. State 1 corresponds to the vehicle being parked at home. State 2 corresponds to the vehicle being on a trip that started from home. State 3 corresponds to the vehicle being parked somewhere else. State 4 corresponds to the vehicle starting a trip from somewhere else than at home. The parameter reduction is thus obtained if it is assumed that the vehicle cannot switch directly from being parked at home to being parked somewhere else, that is from states 1 to 3 . Also it would be reasonable to assume that the vehicle does not drive from home to return to home, without an intermediate stop. Under these assumptions, the matrix of transition intensities becomes:

$\mathbf{Q}(u)=\left(\begin{array}{cccc}-q_{12}(u) & q_{12}(u) & 0 & 0 \\ 0 & -q_{23}(u) & q_{23}(u) & 0 \\ 0 & 0 & -q_{34}(u) & q_{34}(u) \\ q_{41}(u) & 0 & q_{43}(u) & -\left(q_{43}(u)+q_{41}(u)\right)\end{array}\right)$ 
The discrete time transition probability matrix can then be found by (14). In this case the number of parameters to be estimated for each time step is reduced from $N \cdot(N-1)=12$ to 5 , by formulating the model in continuous time as opposed to discrete time. The idea behind this specific model is that it can capture whether the vehicle is parked for different lengths of time, depending on the location. Also it can capture whether the vehicle is usually parked at home at night. As the number of states in the model increases, and supposing that certain structures can be identified, the parameter reduction gained by formulating the model in continuous time is increased.

\section{Hidden Markov Models}

The classical time-varying Markov models only allow for modelling states that are observed. Thus, in this setup, if the data at our disposal is only driving and not driving we are limited to choosing at two-state classical Markov model for describing the data. Furthermore, another important limiting characteristic of time-varying Markov models, is that the time spent in each state is exponentially distributed, albeit with time-varying intensity. This implies that the time until the next transition out of the current state does not depend on the time spent in said state. For models with few states this may be particularly unrealistic.

To address these two important restrictions in a context of limited we introduce a hidden Markov model, which allows us to estimate states that are not directly observed in the data. In such a way that the actual time spent in each observed state is properly captured. A hidden Markov model is obtained by introducing a new state to the original Markov model. The new state is, however, indistinguishable from one or more of the observed states in the original model. This allows for non-exponentially distributed waiting times in each of the observed states, while the Markov assumption is satisfied for the extended model with the hidden states. Specifically the time spent in each observed state is a mixture of exponential distributions. It should be stressed that the same results could be obtained using an ordinary Markov model where the hidden states are actually observed in the data. In short, hidden states are meant to fill the lack of state information. A thorough introduction to hidden Markov models can be found in [33], which also includes R-scripts for parameter estimation.

\section{Parameter Reduction Via B-Splines}

As the number of parameters to be estimated is huge, techniques to reduce this number are needed. One such a technique consists of applying B-splines to approximate the diurnal variation. For a thorough introduction to B-splines as well as other methods for parameter reduction such as smoothing splines and kernels, see [34].

\section{A. B-Splines}

To construct a B-spline, first define the knot sequence $\tau$ such that

$$
\tau_{1} \leq \tau_{2} \leq \cdots \leq \tau_{M}
$$

Let this sequence of knots be defined on the interval where we wish to evaluate our spline. In this particular case the knots should be placed somewhere in the interval $[0,1440]$, that is, over the day.

Denote by $B_{i, m}(x)$ the $i$ th B-spline basis function of order $m$ for the knot sequence $\tau$, where $m<M$. The basis functions are defined recursively as follows:

$$
\begin{aligned}
B_{i, 1}(x) & =\left\{\begin{array}{cc}
1 & \text { if } \tau_{i} \leq x<\tau_{i+1} \\
0 & \text { otherwise }
\end{array}\right. \\
B_{i, m}(x) & =\frac{x-\tau_{i}}{\tau_{i+m-1}-\tau_{i}} B_{i, m-1}(x) \\
& +\frac{\tau_{i}-x}{\tau_{i+m}-\tau_{i+1}} B_{i, m-1}(x)
\end{aligned}
$$

for $i=1, \ldots, M-m$. These basis functions are polynomials of order $m-1$ taking values on the interval $\left[\tau_{1}, \tau_{M}\right]$.

A B-spline curve of degree $m$ is a piecewise polynomial curve defined as follows:

$$
S_{m}(x)=\sum_{i=1}^{M-m} C_{i} B_{i, m}(x),
$$

where $C_{i}, i=\{1, \ldots, M-m\}$, form the control polygon. The $B_{i, m}(x)$ are the B-spline basis functions of order $m$ defined over the knot vector.

As we aim at modeling the diurnal variation in the driving pattern, it is reasonable that the basis splines are periodic. This can be achieved by introducing $2 m$ new knots to the existing knots. The new knots are defined as follows:

$$
\begin{aligned}
\tau_{1-h} & =\tau_{M-h}-\left(\tau_{M}-\tau_{1}\right) \text { for } h \in\{1, \ldots, m\} \\
\tau_{M+h} & =\tau_{h}+\left(\tau_{M}-\tau_{1}\right) \text { for } h \in\{1, \ldots, m\} .
\end{aligned}
$$

More specifically, let the vector containing the new knots be represented by $\tau^{\prime}=\left\{\tau_{1-m}, \ldots, \tau_{M+m}\right\}$. For each B-spline basis function, $m+1$ knots are required, though they may be overlapping. The B-spline basis functions are uniquely defined by the position of the knots. In particular, if the knots are shifted by some constant $\alpha$, the basis functions will be the same as the original, except that they are shifted by $\alpha$. If the new knot vector is defined as $\tau^{\prime}$, the basis function defined by the knots $\left\{\tau_{M}, \ldots, \tau_{M+m}\right\}$ will be the same as that defined for the knots $\left\{\tau_{1-m}, \ldots, \tau_{1}\right\}$, except that it is shifted by the interval length $\tau_{M}-\tau_{1}$. In this way we can define a basis function that is harmonic in the sense that it is recurrent over different days.

All piecewise polynomial splines of order $m$ defined over the knot vector $\tau$ can be constructed from the basis functions defined in (17)-(18). Hence using B-splines does not limit the choice of polynomial splines in any way. Nonetheless, an advantage of using B-splines is that the desired spline can be written as a linear combination of predefined basis functions. This proves useful as a generalized linear model can be applied to estimate the transition probabilities. Traditionally cubic B-splines are used, i.e. $m=4$, which is also the case here. A motivation for using cubic B-splines is that the spline produced will be of order 4 and furthermore, if $\tau_{i} \neq \tau_{j}$ for all $i \neq j$, it will be $\mathcal{C}^{2}$ everywhere. A function which is $\mathcal{C}^{2}$ is indistinguishable from a $\mathcal{C}^{\infty}$ to the human eye. For a further discussion on why to choose cubic splines, see [34]. 


\section{B. A Generalized Linear Model}

To reduce the number of parameters in the model, a Bspline can be fitted to the time-varying transition probabilities $p_{j k}(s)$. There are, however, some issues with this approach. Firstly, there is no guarantee that the fitted B-spline is always in the interval $[0,1]$, which is a problem as we are modeling probabilities. Secondly, if $\sum_{k=1}^{N} n_{j k}(s)=0$ for some $s$, the estimate for $p_{j k}(s)$ given by (5) is undefined. A more refined approach is to use a generalized linear model instead. In the following, such an approach is outlined.

Each day, at a specific minute, a transition from state $j$ to state $k$ either occurs or does not occur. Thus for every $s$ on the diurnal cycle we can consider the number of transitions to be binomially distributed, i.e. $n_{j k}(s) \sim B\left(z_{j}(s), p_{j k}(s)\right)$, where the number of Bernoulli trials at $s$, given by $z_{j}(s)=$ $\sum_{k=1}^{N} n_{j k}(s)$, are known and the probabilities of success, $p_{j k}(s)$, are unknown. The data can now be analyzed using a logistic regression. Using the logit transformation, given by:

$$
\operatorname{logit}(p)=\log \left(\frac{p}{1-p}\right)
$$

the $\log$ odds of the unknown binomial probabilities, $\eta_{j k}(s)$, can be modeled as linear functions of the basis functions $B_{i, m}(s)$, such that:

$$
\begin{aligned}
\eta_{j k}(s) & =\log \left(\frac{p_{j k}(s)}{1-p_{j k}(s)}\right) \\
& =C_{j k, 1} \cdot B_{1, m}(s)+\ldots+C_{j k, M} \cdot B_{M, m}(s)(23)
\end{aligned}
$$

The linear prediction of $\eta_{j k}(s)$ is therefore given by

$$
\widehat{\eta}_{j k}(s)=\widehat{C}_{j k, 1} \cdot B_{1, m}(s)+\ldots+\widehat{C}_{j k, M} \cdot B_{M, m}(s),
$$

where the estimates, $\widehat{C}_{j k, 1}, \ldots, \widehat{C}_{j k, M}$, are found by the iteratively reweighted least squares method.

We can now estimate the the probability of a transition from state $j$ to state $k$ at time $s$ by using the inverse of the logit function, which yields:

$$
\widehat{p}_{j k}(s)=\frac{\exp \left(\widehat{\eta}_{j k}(s)\right)}{1+\exp \left(\widehat{\eta}_{j k}(s)\right)}, \forall j, k .
$$

The procedure of applying a generalized linear model is implemented in the statistical software package $\mathrm{R}$ as the function $\operatorname{glm}(\cdot)$. For a general treatment of this problem see [35].

\section{Choosing the Knots}

Choosing the amount and position of the knots in the knot vector $\tau$ is important to obtain a good fit for the model. A naive method for placing the knots is to distribute them uniformly over the day. A uniform positioning, however, does not take into account the peakedness of the estimate of $p_{j k}(s)$. An algorithm for placing the knots is given in [36].

The proposed algorithm for placing the knots runs as follows:

1) Decide first on the total number of knots, $M$.

2) Decide next on an initial number of knots, $M_{\text {init }}<M$, to be dispersed uniformly in the interval, with one at each endpoint. Denote these knots by $\tau_{\text {init }}$.
3) Fit the model and calculate the likelihood for each knot interval.

4) Find the two adjacent knots with the lowest likelihood of the model in this interval. Denote these knots $\left\{\tau_{j}, \tau_{j+1}\right\}$.

5) Place a new knot, $\tau^{\star}$, in the middle of the interval $\left(\tau_{j}, \tau_{j+1}\right)$.

6) Go to step 3 if the new number of knots $M^{\star}<M$. If $M^{\star}=M$ then stop.

Once an algorithm for distributing the knots is in place, the number of knots to choose, $M$, has to be decided. If the number of knots is too low, the model can be improved by placing additional knots. If the amount of knots is too high, the model is overparameterized. We recommend therefore testing different models recursively up to some large $M$, and choosing the number of knots where there does not seem to be any significant improvement beyond this point.

\section{NUMERICAL EXAMPLE}

In this section the model is fitted to a sample of data collected from the utilization of a single vehicle. The data set solely contains information on whether the vehicle is driving or not driving. To this avail, we introduce hidden driving states to accurately model the duration of the trips. We let the first state denote the vehicle being parked and the others be various driving states. In the estimation procedure we calculate first the off-diagonal elements of $\mathbf{P}(\mathbf{s})$ and then compute the diagonal elements by $\widehat{p}_{i i}(s)=1-\sum_{j=1, j \neq i}^{N} \widehat{p}_{i j}(s)$. Let now $\widehat{p}_{1} \cdot(s)=\sum_{j=1, j \neq i}^{N} \widehat{p}_{i j}(s)$. As we cannot distinguish between the different hidden states, we first determine $\widehat{p}_{1} .(s)$ and then we estimate the probabilities driving the transition into the different hidden states, $\widehat{p}_{1 j}(s)$ for $j \in\{2, \ldots, N\}$, as a fixed proportion of $\widehat{p}_{1} .(s)$. Also $p_{1} .(s)$ is the most interesting parameter, as it is the probability of starting a trip within the next minute, conditional on the vehicle not driving at time $s$.

\section{A. Data}

The example is based on GPS-based data pertaining to a single vehicle in Denmark in the period spanning the five months from 31-10-2002 to 29-03-2003, with a total of 150 days. Our aim is to model the use of this vehicle. The data set only shows whether the vehicle was driving or not driving at any given time. No other information was provided in order to protect the privacy of the vehicle owner. The data set comprises of a total of 799 trips. The time resolution is in minutes.

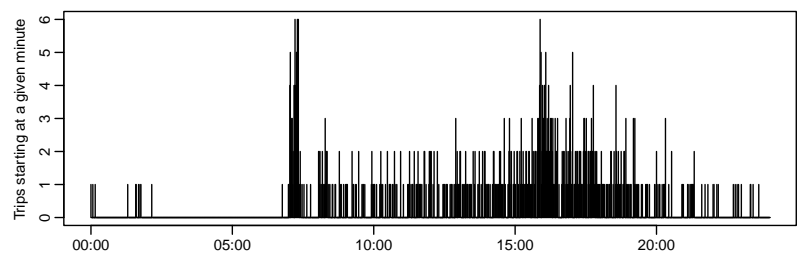

Fig. 1. Trips starting at a certain minute of the day, cumulated for 107 weekdays.

The dataset has been split into two main periods, weekdays and weekends. Figure 1 illustrates the number of trips starting at a given minute for the weekdays. Notice that there is a 
significant degree of diurnal variation, with a lot of trips starting around 07:00 and again around 16:00. Also there are no observations of trips starting between 02:30 and 06:00. Other patterns are found for weekends, but as the approach is similar, we focus on trips starting on weekdays. Driving patterns may also exhibit annual variations, however the limited data sample does not allow for capturing this.

\section{B. Estimation}

Firstly, naive B-splines have been fitted to the data using the logistic regression and the result is shown in Figure 2. These B-splines are described as naive in the sense that the knots defining the basis functions for the B-splines are placed uniformly over the 1-day interval. The gray lines are the estimates $\widehat{p}_{1}$. $(s)$ obtained from (5). As the number of basisfunctions increases, it is apparent that the fit improves.

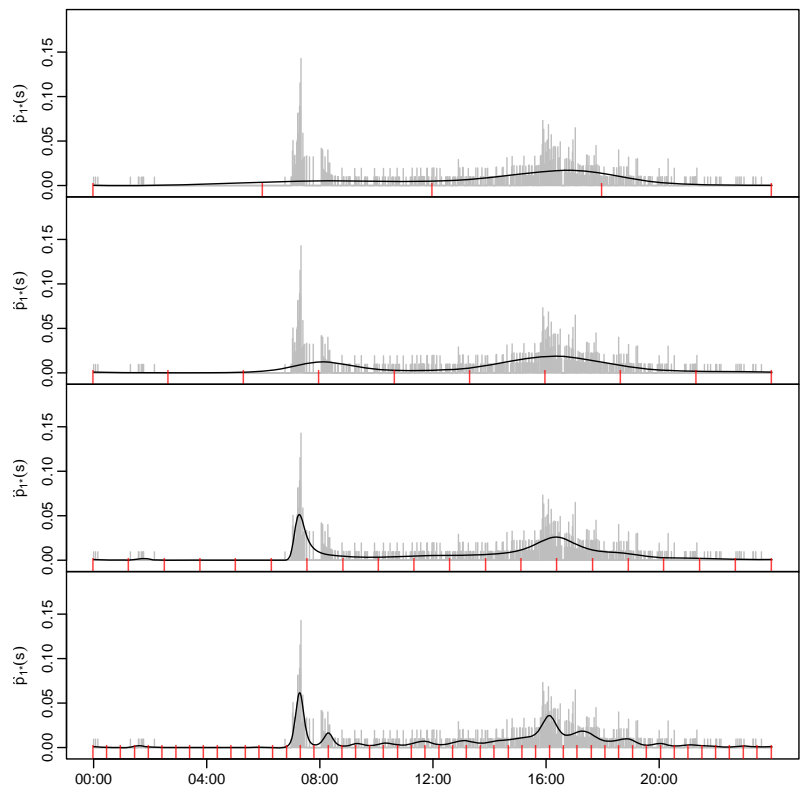

Fig. 2. From top to bottom: Fitting the estimate $\widehat{p}_{1} .(s)$ where the knots are uniformly distributed in the interval from 00:00 to 23:59 on a weekday, with number of knots $\{5,10,20,50\}$. For reference, the gray bars are the estimates of $\widehat{p}_{1} .(s)$ from (5) with no parameter reduction. The red bars indicate the knot positions.

The algorithm for placing the knots is implemented uses an initial amount of knots $M_{\text {init }}=7$. The left plot in Figure 3 shows the tests for different number of knots, $M_{n}$.
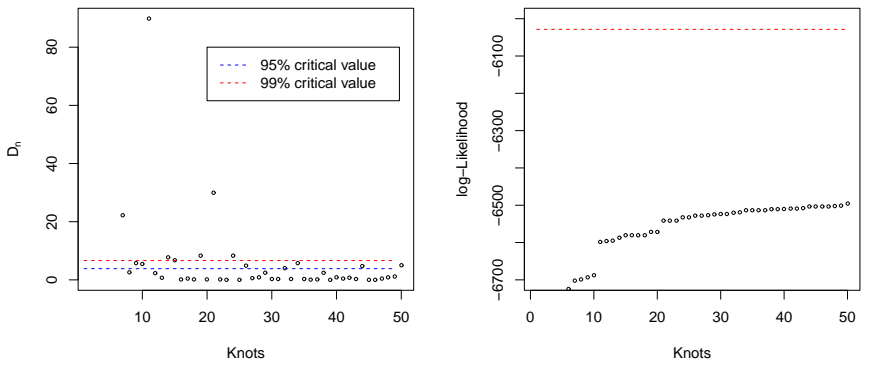

Fig. 3. Left: Log-likelihood ratio test statistic, given by $D_{n}$, from the model with $n$ knots vs. the model with $n-1$ knots. $95 \%$ and $99 \%$ critical values are shown for a $\chi^{2}$-distribution with one degree of freedom. Right: The log-likelihood of the models with different knots. The red dashed line is the likelihood of the model with estimates based on (5).
Referring to Figure 3, left, the model with a total number of knots $M_{n}=21$ is chosen, as no significant improvement is attained beyond this point. In Figure 3, right, the log-likelihood for models with different numbers of knots is shown. The red dashed line is the log-likelihood of the model with the estimates found by (5) and corresponds to a perfect data fit. It is in some sense a limit for the fitted models.

The models based on B-splines are sub-models of the model in which a knot is placed at every minute. In this model, the transition probabilities are estimated independently for every minute, and in turn the model corresponds to that with no parameter reduction. The models where the number of parameters is reduced can be tested against the model with no parameter reduction. This leads to a test statistic that will be $\chi^{2}$-distributed with $1440-M$ degrees of freedom for each time-varying transition probability. Accordingly the critical value will be very large ( $>1475$ for estimating one timevarying transition probability for $M \leq 50$ at $95 \%$ significance) and thus a test for sufficiency is not appropriate.

The top plot in Figure 4 illustrates the estimate of $\widehat{p}_{1} .(s)$ using B-splines with $M=21$, where the knots are placed by the algorithm introduced in Section 3.3. For comparison, the model with the naive knots and $M=21$ is shown on the bottom plot in Figure 4. By visual inspection, it is observed that the model in which the knots are placed according to the algorithm in Section 3.3 better captures the peakedness of $p_{1} \cdot(s)$.

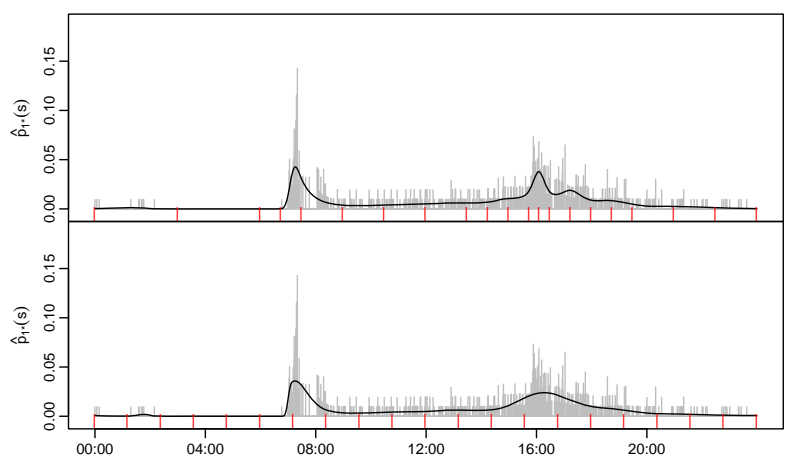

Fig. 4. Top: $\widehat{p}_{1} .(s)$ based on the B-splines with $M=21$ and the knots placed using the algorithm, plotted as the black line over the estimates $\widehat{p}_{1} .(s)$ with no parameter reduction. Bottom: $\widehat{p}_{1} .(s)$ based on the naive B-splines with $M=21$, plotted as the black line. The red bars indicate the knot position.

From the estimation of the transition probabilities and the evaluation of likelihoods we conclude that a Markov model including five hidden time-homogeneous driving states and one inhomogeneous non-driving state satisfactorily describes the use of the vehicle. Indeed, with such a number of states, we manage to properly capture the trip lengths. In Figure 5 the empirical trip lengths obtained from the data are plotted along with the distribution of the trip lengths based on the fitted model. Notice that the distribution is clearly not exponential.

\section{Applications}

The applications of the proposed stochastic model for driving patterns range from simulating different driving scenarios to calculating the probability of a trip starting within a given 


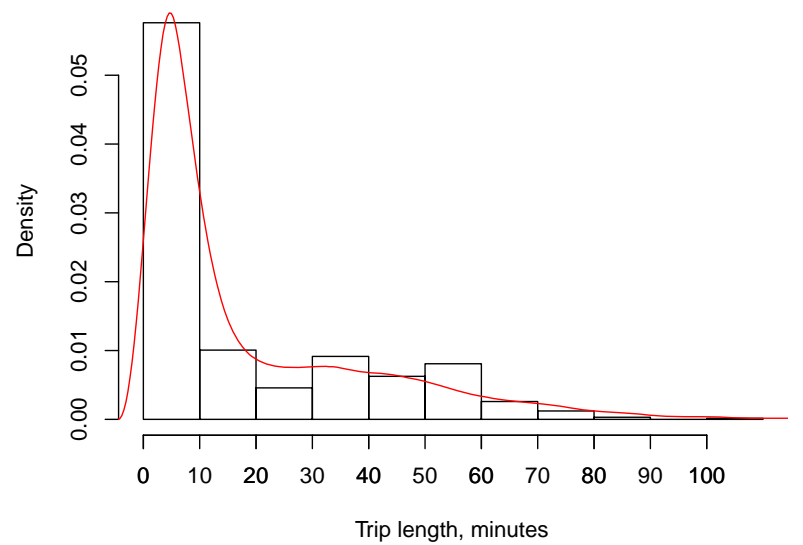

Fig. 5. Trip lengths, empirical in histogram bars, and estimated density, in red, based on Monte Carlo simulation from fitted model.

interval. In addition, the model is prerequisite to determine the optimal charging scheme for an electric vehicle.

1) Probabilities and Simulations: Four driving scenarios are simulated and shown in Figure 6. Markov states are indicated in a binary form depending on whether the vehicle is driving " 1 " or not " 0 ".

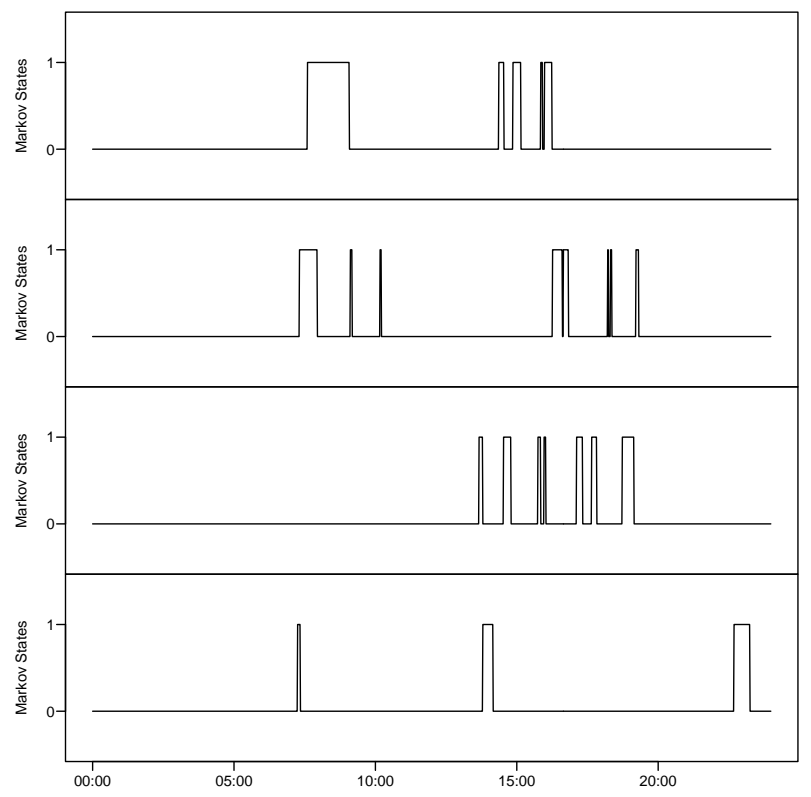

Fig. 6. Four distinct realizations of driving patterns using the proposed stochastic model.

Next we illustrate how to find the probability of a trip starting within a given interval. This probability is to be consider as probability under the model. That is given the model assumptions and the parameter estimates are correct. Suppose that at time $s$ the vehicle is parked. Denote the waiting time until the next trip starts by $Z_{s}$. We have that $Z_{s} \sim \exp \left(q_{1} .(s)\right)$, where $q_{1} .(s)=\sum_{j=2}^{N} q_{1 j}(s)$. The probability of a trip within the time interval $[s, s+\tau]$ is thus

$$
\mathbb{P}\left(Z_{s} \leq \tau\right)=1-e^{-\int_{0}^{\tau} q_{1} \cdot(s+t) d t} .
$$

Using this equation, for example, the probability of a trip starting in the interval from 00:00 to 06:00 is $\mathbb{P}\left(Z_{00: 00} \leq\right.$ $06: 00)=1-e^{-\int_{0}^{6 \cdot 60} q_{1 \cdot}(t) d t}=0.098$.

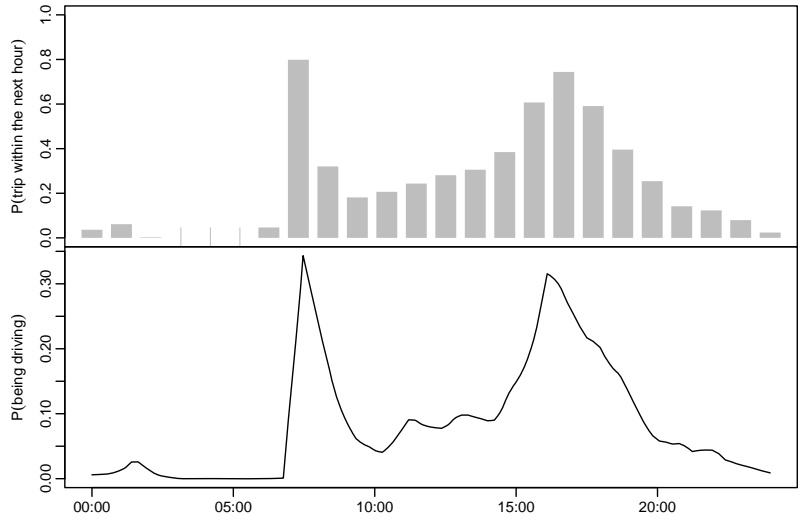

Fig. 7. Top: A graph plotting the probability of starting a trip within the next hour, conditional on not driving at the present time, which is found by applying (26). Bottom: The probability of the vehicle being in use at any time of the day, which is estimated using bootstrap.

In the top part of Figure 7, the probability of starting a trip within the next hour, conditional on not driving at the beginning of that hour, is depicted. The probability of the vehicle being in use at any time of the day is found using bootstrap ([37]) and is shown in the bottom part of Figure 7.

2) Example of Electric Vehicle Charging: In [27] the model for driving patterns, developed in this paper, is used as an input into an optimization problem, with the objective of minimizing charging costs and user inconvenience. They show that the randomness intrinsic to driving behavior has a substantial impact on the charging strategy to be implemented. This holds true both in terms of savings and in terms of satisfying the driving needs. It is especially the case for vehicle to grid schemes, where the knowledge of the users driving needs are essential to ensure a sufficient charge level for the users trip.

\section{Conclusion and Future Research}

This paper proposes a suitable model that captures the diurnal variation in the use of a vehicle. The number of parameters is significantly reduced by using B-spline basis functions as explanatory variables in a logistic regression. The model is versatile and can be applied to describe driving data from any single vehicle, thus providing a reliable model for the use of that vehicle.

It would be interesting to apply the model to data that includes location, to see how this affects the model. The model could be extended to cover a population of vehicles by using a mixed-effect model. Another extension to the model could be to estimate the transition probabilities adaptively in time. This way structural changes in the driving behaviour of the vehicle user, such as variation over the year or a change in use as a result of from the household purchasing an additional vehicle, could be captured. An obvious next step is to use the model to implement a charging strategy that minimizes the costs of driving considering the underlying uncertainty in the use of the vehicle.

\section{REFERENCES}

[1] H. Morais, T. Sousa, Z. Vale, and P. Faria, "Evaluation of the electric vehicle impact in the power demand curve in a smart grid environment," Energy Conversion and Management, vol. 82, pp. 268-282, 2014. 
[2] C. Weiller, "Plug-in hybrid electric vehicle impacts on hourly electricity demand in the united states," Energy Policy, vol. 39, no. 6, pp. 37663778, 2011.

[3] M. A. Ortega-Vazquez, F. Bouffard, and V. Silva, "Electric vehicle aggregator/system operator coordination for charging scheduling and services procurement," Power Systems, IEEE Transactions on, vol. 28, no. 2, pp. 1806-1815, 2013.

[4] B.-G. Kim, S. Ren, M. van der Schaar, and J.-W. Lee, "Bidirectional energy trading and residential load scheduling with electric vehicles in the smart grid," Selected Areas in Communications, IEEE Journal on, vol. 31, no. 7, pp. 1219-1234, 2013.

[5] S. Habib, M. Kamran, and U. Rashid, "Impact analysis of vehicle-to-grid technology and charging strategies of electric vehicles on distribution networks-a review," Journal of Power Sources, vol. 277, pp. 205-214, 2015.

[6] M. J. Kofler, C. Reinisch, and W. Kastner, "A semantic representation of energy-related information in future smart homes," Energy and Buildings, vol. 47, pp. 169-179, 2012.

[7] A. J. Collin, G. Tsagarakis, A. E. Kiprakis, and S. McLaughlin, "Multi-scale electrical load modelling for demand-side management," in Innovative Smart Grid Technologies (ISGT Europe), 2012 3rd IEEE PES International Conference and Exhibition on. IEEE, 2012, pp. 1-8.

[8] P. Samadi, H. Mohsenian-Rad, R. Schober, and V. W. Wong, "Advanced demand side management for the future smart grid using mechanism design," Smart Grid, IEEE Transactions on, vol. 3, no. 3, pp. 1170$1180,2012$.

[9] A.-H. Mohsenian-Rad, V. W. Wong, J. Jatskevich, R. Schober, and A. Leon-Garcia, "Autonomous demand-side management based on game-theoretic energy consumption scheduling for the future smart grid," Smart Grid, IEEE Transactions on, vol. 1, no. 3, pp. 320-331, 2010.

[10] M. D. Galus, M. G. Vayá, T. Krause, and G. Andersson, "The role of electric vehicles in smart grids," Wiley Interdisciplinary Reviews: Energy and Environment, vol. 2, no. 4, pp. 384-400, 2013.

[11] T. Golob and J. Gould, "Projecting use of electric vehicles from household vehicle trials," Transportation Research Part B: Methodological, vol. 32, no. 7, pp. 441-454, 1998.

[12] N. Pearre, W. Kempton, R. Guensler, and V. Elango, "Electric vehicles: How much range is required for a day's driving?" Transportation Research Part C: Emerging Technologies, vol. 19, no. 6, pp. 1171-1184, 2011.

[13] R. Green, L. Wang, and M. Alam, "The impact of plug-in hybrid electric vehicles on distribution networks: a review and outlook," in Power and Energy Society General Meeting, 2010 IEEE, july 2010, pp. 1 -8.

[14] N. Rotering and M. Ilic, "Optimal charge control of plug-in hybrid electric vehicles in deregulated electricity markets," Power Systems, IEEE Transactions on, vol. 26, no. 3, pp. 1021 -1029, aug. 2011

[15] S. Acha, T. C. Green, and N. Shah, "Effects of optimised plug-in hybrid vehicle charging strategies on electric distribution network losses," in Transmission and Distribution Conference and Exposition, 2010 IEEE PES, april 2010, pp. $1-6$.

[16] L. Gan, U. Topcu, and S. Low, "Optimal decentralized protocol for electric vehicle charging," California Institute of Technology, Tech. Rep., 2011. [Online]. Available: http://www.cds.caltech.edu/ utopcu/images//9/96/GTL-cdc11.pdf

[17] A. Lojowska, D. Kurowicka, G. Papaefthymiou, and L. van der Sluis, "From transportation patterns to power demand: Stochastic modeling of uncontrolled domestic charging of electric vehicles," in Power and Energy Society General Meeting, 2011 IEEE, july 2011, pp. 1 -7.

[18] Z. Ma, D. Callaway, and I. Hiskens, "Decentralized charging control for large populations of plug-in electric vehicles: Application of the Nash certainty equivalence principle," in Control Applications (CCA), 2010 IEEE International Conference on, sept. 2010, pp. $191-195$.

[19] P. Richardson, D. Flynn, and A. Keane, "Optimal charging of electric vehicles in low-voltage distribution systems," Power Systems, IEEE Transactions on, vol. 27, no. 1, pp. 268 -279, feb. 2012.

[20] R. Bessa, F. Soares, J. Pecas Lopes, and M. A. Matos, "Models for the ev aggregation agent business," in PowerTech, 2011 IEEE Trondheim. IEEE, 2011, pp. 1-8.

[21] P. Grahn, K. Alvehag, and L. Soder, "Plug-in-vehicle mobility and charging flexibility Markov model based on driving behavior," in European Energy Market (EEM), 2012 9th International Conference on the. IEEE, 2012, pp. 1-8.

[22] G. Hill, P. Blythe, and C. Higgins, "Deviations in Markov chain modeled electric vehicle charging patterns from real world data," in Intelligent Transportation Systems (ITSC), 2012 15th International IEEE Conference on. IEEE, 2012, pp. 1072-1077.
[23] J. Rolink and C. Rehtanz, "Estimation of the availability of gridconnected electric vehicles by non-homogeneous semi-Markov processes," in PowerTech, 2011 IEEE Trondheim. IEEE, 2011, pp. 1-7.

[24] F. Soares, J. P. Lopes, P. R. Almeida, C. Moreira, and L. Seca, "A stochastic model to simulate electric vehicles motion and quantify the energy required from the grid," in 17th Power Systems Computation Conference, 2011, pp. 22-26.

[25] G. Souffran, L. Miegeville, and P. Guerin, "Simulation of real-world vehicle missions using a stochastic Markov model for optimal design purposes," in Vehicle Power and Propulsion Conference (VPPC), 2011 IEEE. IEEE, 2011, pp. 1-6.

[26] A. Schlote, E. Crisostomi, S. Kirkland, and R. Shorten, "Traffic modelling framework for electric vehicles," International Journal of Control, vol. 85, no. 7, pp. 880-897, 2012.

[27] E. B. Iversen, J. M. Morales, and H. Madsen, "Optimal charging of an electric vehicle using a markov decision process," Applied Energy, vol. 123, pp. 1-12, 2014.

[28] R. Romo and O. Micheloud, "Power quality of actual grids with plug-in electric vehicles in presence of renewables and micro-grids," Renewable and Sustainable Energy Reviews, vol. 46, pp. 189-200, 2015.

[29] R. Liu, L. Dow, and E. Liu, "A survey of pev impacts on electric utilities," in Innovative Smart Grid Technologies (ISGT), 2011 IEEE PES. IEEE, 2011, pp. 1-8.

[30] M. Yilmaz and P. T. Krein, "Review of the impact of vehicle-togrid technologies on distribution systems and utility interfaces," Power Electronics, IEEE Transactions on, vol. 28, no. 12, pp. 5673-5689, 2013.

[31] G. Grimmett and D. Stirzaker, Probability and Random Processes, ser Texts from Oxford University Press. Oxford University Press, 2001. [Online]. Available: http://books.google.dk/books?id=G3ig-0M4wSIC

[32] Y. Pawitan, In All Likelihood: Statistical Modelling and Inference using Likelihood, ser. Oxford science publications. Clarendon Press, 2001. [Online]. Available: http://books.google.dk/books?id=M-3pSCVxV5oC

[33] W. Zucchini and I. MacDonald, Hidden Markov Models for Time Series: An Introduction Using $R$, ser. Monographs on Statistics and Applied Probability. Taylor \& Francis Group, 2009. [Online]. Available: http://books.google.dk/books?id=LDDzvCsdVs8C

[34] T. Hastie, R. Tibshirani, and J. Friedman, The Elements of Statistical Learning: Data Mining, Inference, and Prediction, ser. Springer series in statistics. Springer, 2008. [Online]. Available: http://books.google.dk/books?id=tVIjmNS3Ob8C

[35] H. Madsen and P. Thyregod, Introduction to General and Generalized Linear Models, ser. Chapman \& Hall/CRC Texts in Statistical Science. Chapman \& Hall/CRC, 2010. [Online]. Available: http://books.google.dk/books?id=JhuDNgAACAAJ

[36] W. Mao and L. H. Zhao, "Free-knot polynomial splines with confidence intervals," Journal of the Royal Statistical Society: Series B (Statistical Methodology), vol. 65, no. 4, pp. 901-919, 2003. [Online]. Available: http://dx.doi.org/10.1046/j.1369-7412.2003.00422.x

[37] A. Davison and D. Hinkley, Bootstrap Methods and Their Application, ser. Cambridge Series in Statistical and Probabilistic Mathematics. Cambridge University Press, 1997. [Online]. Available: http://books.google.dk/books?id=4aCDbm_t8jUC 